\title{
Building Automation for Increased Energy Efficiency in Buildings
}

\author{
Gerhard Zucker \\ Austrian Institute of Technology \\ Energy Department \\ gerhard.zucker@ait.ac.at
}

\author{
Tarik Ferhatbegovic \\ Austrian Institute of Technology \\ Energy Department \\ tarik.ferhatbegovic.fl@ait.ac.at
}

\author{
Dietmar Bruckner \\ Institute of Computer Technology \\ Vienna University of Technology \\ bruckner@ict.tuwien.ac.at
}

\begin{abstract}
The implementation of components and systems of building automation, in particular for HVAC systems and renewables, opens the possibility to increase the energy efficiency of (clusters of) buildings even further than originally intended. By additionally implementing different control strategies, it is possible to use the thermal storage capacity of buildings to improve their electrical characteristic in order to better meet the needs of the electricity grid. However, it is usually hard to obtain the thermal storage parameters of a building, because they need to be computed in complex thermal simulations. In this paper we present a way to quickly estimate an approximation thereof and feed it to strategies of different complexity, dependent on the free resources of the building automation installation.
\end{abstract}

\section{INTRODUCTION}

Building automation today provides the possibility to control functional buildings with different level of details [1]: room controllers allow maintaining indoor comfort in offices, control strategies are executed on automation level in substations and a building management system allows the building operator to get a complete overview of the building and its systems. While every manufacturer has its own solution to building operation, the general understanding is that it needs to be robust and maintain indoor comfort even during particularly hot or cold days. Therefore control strategies are designed in a way that will guarantee permanent comfort. Preferably the control strategies remain constant, possibly alternating between winter and summer operation, but without dynamic changes on a regular, e.g. daily, basis. The reason can be seen in the above mentioned robustness in order to guarantee constant comfort.

Looking from a different perspective, an automated building is a system that provides three interesting properties: available degrees of freedom, considerable thermal storages and the ability to control the whole system using methods of information technology. This paper examines the possibilities of exploiting the degrees of freedom for the sake of optimizing energy efficiency. We propose three different approaches, examine their advantages and disadvantages and prove their feasibility with thermal simulations of existing buildings (see also [2]).

The rest of this paper is organized as follows: chapter II gives an introduction about the three main goals of energy efficiency by adjusting the control strategies of the premises' HVAC system (heating, ventilation, air condition) and renewables. Next, a quick first order approximation of the dynamic thermal building behavior is presented in chapter III.
Chapters IV and V describe two ways of using the thermal model, a parameter optimization strategy and model predictive control, respectively. Finally, Chapter IV sums up and concludes the paper.

\section{ENERGY EFFICIENCY AND BUILDING CONTROLS}

Energy supply systems in a modern building have a considerable degree of complexity. Their design is influenced by different life cycle phases (e.g. planning and operation), industries (e.g. HVAC, shading and safety) and media (e.g. hot water and electricity). Building automation has significant impact on energy efficiency, which is by now also reflected in standardization activities e.g. in EN 15232 [3]. Installing and commissioning building controls is usually the last step in a long chain of phases during building construction and is done at the very end of the building phase (and usually recalibrated after some time of operation). Therefore, the first goal of controls is to get the building into operation and maintain indoor comfort. Given the limited time in this phase (between construction and operation) the solutions have to be based on existing templates and can only be slightly adapted to the system at hand.

The control strategies in thermal systems like heating or cooling do not have strong requirements with regard to system dynamics: the processes in a building are very slow and are always lossy, which reduces their tendency to oscillate. Controls are linear, i.e. using P- or PI-controllers, although the system is often operated broadband where the linear model is not valid anymore. In order to prevent malfunction, the controls are therefore configured to be on the safe side: very slow dynamics in the linear controllers (i.e. small proportional and integral actions) to avoid oscillations in the presence of big delays (e.g. due to mass transport in long pipes), time schedules that guarantee the expected comfort at a certain time (by preheating or precooling well in advance) and set-points that ensure comfort even in the most remote room with the biggest losses to the outside.

Due to reasons of reliability and safety buffers, energy systems are usually overengineered in order to deliver heating, cooling or air volume even if the building physics does not meet the original construction plan.

All in all an automated building provides a control system that allows fine-grained control of energy consumption, energy systems that have the potential to provide flexibility in their operation, and a building that has the capability of storing thermal energy in its internal structures (e.g. concrete 
walls, floors and ceilings). The building creates a consumption profile (and a production profile, given that renewable energy sources like photovoltaic or solar thermal systems are available) for electric energy (and thermal energy, if it consumes thermal energy from district heating). Today these consumption profiles are only determined by the internal processes of the building: heating, cooling, lighting, and internal loads. While some of the consumers like lighting are directly tied to user comfort and cannot easily be influenced, other consumers that are related to thermal processes can be modulated without the user noticing. These processes provide us with the necessary degrees of freedom for optimization.

\section{A. Energy Efficiency}

There are three main starting points for optimization with regard to energy processes, while the overall goal is to increase energy efficiency. This includes simple solutions like monitoring energy consumption in the different systems and detecting improvements, but also faults in the system (e.g. heating and cooling demand at the same time in different areas of the building, systems that are permanently running, broken valves, etc.). More complex approaches are described in the following chapters.

\section{B. Shaping the Electric Load Profile}

The second goal is the modification of the consumption profile. Today only the electric profile is of interest, because research on the thermal consumption profile (taken from the district heating network) is only at the beginning of exploiting flexibilities [4]. In the electric grid the building can be changed from a passive consumer into an active participant by modifying the consumption profile of selected consumers. This is an important contribution of turning the existing grid into a smart grid, which unites energy and information technology; other contributions to this development can be found in [5] and [6]. As said above, electric consumers involved in heating, cooling and ventilation are of main interest, lighting or the control of white goods pose more problems and less potential. The shaping of electric load profiles has two benefits: reduction of greenhouse gases and the possibility of cost savings for the end user. Seeing the current development of electricity consumption it is clear that the electric grid is about to reach its limits with regard to peak load capacity. During the morning and the evening electric consumption is considerably high; any reduction of electric consumption supports the grid and reduces the risk of power shortages. As soon as tariffs are available that let the user benefit from exploiting the possibilities to shift electric load, the user can reduce energy costs and thus benefits from the flexibility of the building. Furthermore, while band production of electricity is more environmental-friendly (e.g. hydropower plants), peak production has to be covered by fossil energy like gas turbines and thus increases the overall greenhouse gas emissions. Thus, reduction of peak load has a double benefit both for the electric grid and the carbon footprint (see also [7]).

\section{Integration of Renewable Energy Sources}

Just like the consumption of electricity can be shaped to fit the requirements of the electric grid, it can be adapted to support another goal, which additionally also supports the grid: when a photovoltaic system is installed on the building, electricity is produced according to the available sun radiation. Especially in residential buildings it is possible that the peak production (around noon) is bigger than the overall electrical consumption. The excess energy is fed back into the grid, resulting in additional load for the electric grid (this time in the other direction). Since the utility company needs to maintain a voltage level that must not exceed an upper boundary, a larger amount of photovoltaic systems can cause problems in the grid. Depending on the tariff scheme, feeding back electricity into the grid is not as cost-effective as consuming electricity directly in the building, and therefore a disadvantage for the building owner. Finally, increasing the production of renewable energy by photovoltaic systems is vital for maintaining energy supply in the near future, but must be achieved without causing problems in the electric grid.

\section{Building simulation}

We see that a building has multiple ways to be optimized. First, the overall efficiency can be improved by taking a closer look at the existing control strategies, then the thermal processes can be exploited to shift electric load in order to achieve optimization goals like peak load reduction or maximum consumption of self-produced electricity.

Before these degrees of freedom can be exploited it is necessary to have an understanding of the system that is optimized. This is done by creating a physical model of the building and its energy systems. Common programs for thermal simulation are TRNSYS [8] or EnergyPlus [9], complex simulations including electric consumption can be programmed in the object oriented modeling language Modelica [10], with Dymola [11] being a software tool that supports Modelica. A thermal building model describes the dynamic thermal behavior with regard to losses through the envelope, gains by solar radiation and internal loads and derives the temperature distribution in the different thermal zones of a building. The energy systems of the building (heating, cooling, ventilation) modify the simulated indoor climate and can be modeled as simple energy sources (e.g. contribute the amount of energy that was lost during one simulation step so that the building maintains its equilibrium) or they can themselves have dynamic properties; also the controls can be included into the simulation (e.g. a PIcontroller maintaining room temperature). Using this model the electric consumption of the HVAC components can be simulated and thus the part of the electric consumption profile that can be influenced. By modifying the schedules and control strategies of heating, cooling and ventilation the electric profile can be adapted to meet the optimization goals. The following chapters describe three approaches how to 
achieve the different optimization goals; the complexity and modeling effort rises with each of the approaches.

\section{First ORder APPROXIMATION OF BUILdING Physics}

In order to facilitate load shifting of HVAC components it is important to know how the building, especially the room temperature, responds to stopping the supply with heating or cooling energy. In any case the comfort boundaries will be violated after some time and it is important to find the worst case, i.e. the shortest time when the building reaches a boundary. This is the time when the energy systems have to be switched on again to restore indoor comfort; it is also the longest possible duration for load shifting.

For this situation the thermal simulation of the building can be reduced to modeling the heating up and cooling down of the building. The approach sketched in [2] and described in more detail in [12] and [13] and creates a first order thermal model, reducing the thermal simulation to a first order differential equation and thus a single time constant for cooling down and one for heating up. The differential equation is solved by an exponential function, shown for cooling in equation (1); $T_{\text {ronm }}$ is the indoor room temperature, $T_{\text {start }}$ the start temperature before the cooling process starts, $T_{\text {outside }}$ is the outside temperature, and $\tau_{\text {conl }}$ the time constant of the building.

$$
T_{\text {room }}=\left(T_{\text {start }}-T_{\text {outside }}\right) \cdot e^{-t / \tau_{\text {cool }}}+T_{\text {outside }}
$$

The time constant $\tau_{\text {rool }}$ is the building specific, first-order approximation, which is an envelope for all thermal effects in one single constant. Deriving this constant is the crucial point in time efficient monitoring. The first order approximation is intended for situations, where a full-fledged thermal simulation is not possible, e.g. when the buildings of a district or a whole city shall be modeled. In this case it is important to get the time constant with as little effort as possible. While thermal building models do not take too many resources when running a simulation, the creation of thermal building models is quite tedious, since the geometry and all the materials have to be created in a 3D modeling tool. Within the project Building2Grid (funded by the Austrian Research Promotion Agency, project nr. 825545) a total of ten buildings in Salzburg, Austria, are examined in order to derive the time constants for heating and cooling. The buildings are therefore simulated in TRNSYS (Fig. 1 showing a Google SketchUp [14] model of a building that is imported into TRNSYS).

The simulation shows the building dynamics when the heating supply is switched off. The resulting cool-down is then approximated by an exponential function, resulting in the time constant shown in Table I. In this table the simulation results for five of the ten buildings are listed, together with their specific annual heat energy demand. For better understanding the last column shows the timespan it takes the indoor temperature to drop for $2{ }^{\circ} \mathrm{C}(3.6 \mathrm{~F})$. The simulations were done with an air exchange rate of 0.5 per hour (i.e. in one hour half of the total air volume is exchanged with outdoor air, which is a common value for residential buildings) and an outside temperature of $-12{ }^{\circ} \mathrm{C}(10.4 \mathrm{~F})$.

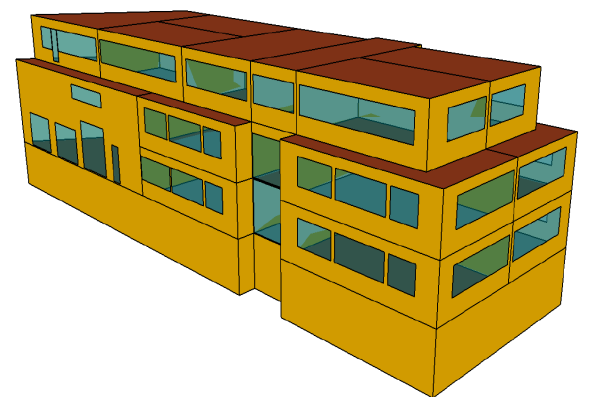

Fig. 1. Building model for thermal simulation in TRNSYS. The model was created in Google Sketchup.

The method is useful for large scale approximations of load shifting potentials. When upscaling it to district level or even city level, the TRNSYS simulation, which has been used here to create the time constants, can be omitted, thus saving the effort for creating the building models. Instead, the time constants are derived from existing building data. Today many buildings have an energy performance certificate that contains all the necessary data about the building structure to quickly derive the necessary cooling-down and heating-up dynamics. Strictly speaking the equivalent time constant $\tau_{\text {conl, }}$, which includes all (nonlinear) effects of heat radiation, convection, and conduction depends on multiple parameters and is therefore not exactly a constant. Most importantly, the cooling-down strongly depends on the air exchange rate, which is one of the parameters in $\tau_{\text {cool }}$. Still the method is an important step to estimate the thermal building dynamics that are needed to exploit intrinsic thermal storages in buildings.

TABLE I

COOLING CASE OF 5 BUILDINGS IN SALZBURG, AUSTRIA

\begin{tabular}{|c|c|c|c|c|}
\hline $\begin{array}{l}\text { Building } \\
\text { type }\end{array}$ & $\begin{array}{l}\text { Year of } \\
\text { construction }\end{array}$ & $\begin{array}{l}\text { Specific annual } \\
\text { heat energy } \\
\text { demand } \\
{\left[\mathrm{kWh} / \mathrm{m}^{2} \mathrm{a}\right]}\end{array}$ & $\begin{array}{l}\text { Time } \\
\text { constant } \\
\text { cooling } \\
{[\mathrm{h}]}\end{array}$ & $\begin{array}{l}\text { Cooldown } \\
\text { from 22 to } \\
20^{\circ} \mathrm{C}[\mathrm{h}]\end{array}$ \\
\hline Residental & 1992 & 104 & 122 & 7,3 \\
\hline Residental & 1994 & 75 & 124 & 7,5 \\
\hline Residental & 1994 & 108 & 86 & 5,2 \\
\hline Residental & 2003 & 35 & 128 & 7,7 \\
\hline Office & 2009 & 20 & 206 & 12,4 \\
\hline
\end{tabular}

\section{IV.OPTIMIZATION OF EXISTING CONTROL STRATEGIES}

Building automation on field level and automation level relies on a mix between linear controllers (mainly P- and PIcontrollers; PID-controllers are hardly used due to the relaxed dynamics in buildings) on field level and control strategies on automation level that are described in sequences of states. Until some ten years ago these states were programmed in different programming languages e.g. Structured Text, a language for Programmable Logic Controllers (PLCs), which is part of the IEC 61131-3 standard [15]. While PLCs are still 
used in some systems, it is now more common to use graphical, block-based programming that is done by selecting predefined function blocks and connecting the inputs and outputs of the blocks. This way the creation of control strategies is reduced to selecting and connecting predefined (and pre-tested) blocks and parameterizing the blocks. Such a measure reliefs the programmer of debugging large amounts of code lines and reduces the likeliness of errors. There is usually a toolchain available that allows compiling and downloading the control strategies to the automation station, where it is executed during operation. The whole process of graphical programming speeds up the design and creation of control strategies and it also allows testing the program on a high semantic level. For off-site testing it is possible to simulate inputs and check the according behavior of the program. This allows to start commissioning with a program that is syntactically correct, but also can be checked for its basic semantics with a set of test inputs.

Research takes advantage of the high semantic level of graphical programming by optimizing a few relevant parameters without the need to work with actual code - which would require much more complex analysis. The control strategies have some fundamental elements like criteria to switch a component on or off or set-points that a component needs to reach (e.g. by using a feedback controller). Modeling effort is reduced to a level of energy balances, where components produce or consume the expected amount of energy. The approach in project KOMBINE (funded by the Austrian Research Promotion Agency, project nr. 829718) is to model, simulate and optimize the control strategies of a pellets-stove with a storage tank that is supported by a solar thermal system. The stove produces domestic hot water for heating and tap water and the optimization goal is to reduce the biomass contribution and maximize usage of the solar thermal system. To do so a model of the control strategies is created that reflects the different states of operation e.g. igniting the pellets or running the solar thermal pump. The challenge is to model the physical system in a way that allows to derive production of hot water. Also the consumption is a critical factor, since the storage tank only has limited capacity: once the temperature in the tank is too low the user comfort would be violated, which has to be prevented by heating up the pellets stove. All system constraints and the physical properties of pellets stove and solar thermal system are modeled using TRNSYS and Matlab. The conditions for activating the pellets stove or the solar thermal pump as well as several set-points for the controlled system are determined from the solution of an optimization problem. Based on an objective function that regards usage of pellets and electricity consumption, the algorithm can optimize the simulated system based on a consumption profile that reflects average usage during winter, summer and the transitional periods. In order to save simulation time, not a whole year is simulated, but rather a selection of design days, reflecting the typical outside conditions for a geographical location (in this case for Austria, Europe). Selecting representative design days instead of simulating all days of a year is a common methodology in thermal simulation. The models that are used for the oven and the solar thermal system are validated in a test rig, so that the following optimization can rely on data about thermal production of pellets and solar thermal system. It is expected to get an improved set of parameters for the control strategy and possibly also recommendations on how to improve the system architecture.

\section{Model-BASEd Predictive CONTROL}

Model-based predictive control (MPC) concepts have gained popularity within the research community as well as industry addressing different fields over the last decades when model based predictive control first appeared in the late 1970s. In the area of building and buildings system control, MPC offers a good possibility to pursue the energy efficient control on the one hand and to deal with the slow thermal dynamics and system stiffness (i.e.: due to different dynamics within the controlled system) as well as time delays (i.e.: due to mass transport) on the other hand. MPC is finding recognition within building research increasingly.

The energy efficient application of MPC in buildings has been successfully proven in e.g.: [16] and [17]. The authors of [17] report on theoretical energy savings between $16 \%$ and $41 \%$ employing MPC concepts. Some other works such as [18] take explicitly advantage of weather forecast information and stochastic MPC to improve the energy efficient operation in room automation. The work [19] addresses several MPC strategies to perform HVAC system control to achieve higher thermal comfort and optimal HVAC system operation in the first place.

The idea of the MPC approach is to use a physical model of the system to be controlled along with a predefined objective function which is minimized to obtain the (optimal) control signal acting on the real system. Hereby, the model of the controlled system serves as the basis to obtain the approximate future dynamic behavior of the system being subject to control. In order to account for the non-negligible model-plant mismatch, the receding horizon scheme is commonly employed allowing for the consideration of instantaneous state and disturbance information (including varying solar radiation and weather conditions in present and future as described in [20]), thus providing the possibility to re-run the optimization routine and reduce the effect of model-plant mismatch. Fig. 2 shows the basic principle of the predictive control approach.

An appropriate model of the system providing the information about system dynamics is a prerequisite for the actual controller design. There exists a variety of different approaches to system modelling suitable for the design of the predictive controllers in particular, see e.g.: [7]. State-space representation of the modelled system (e.g.: by using first principle approaches) is a common way to describe the physical system behaviour and to serve as the basis for the subsequent procedure of designing the predictive controller. 
In general, the distinction is made between linear and nonlinear model based predictive control approaches. The former use a linear representation of the real system, therefore, usually addressing only a small proximity of the desired operating point. The latter, however, capture systeminherent nonlinearities, allowing for the operation in a wide region around the desired operating point. This, in turn, is an asset, considering the fact that energy systems are often forced to operate away from the envisaged point of operation (e.g.: operating point of the system is shifted due to weather changes).

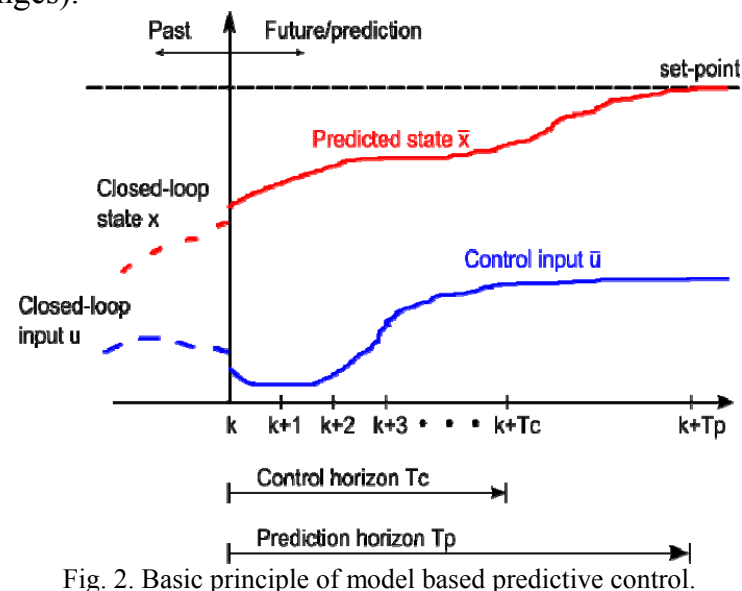

Linear MPC shows advantages over the nonlinear approach mainly with regards to the computational time. Usually the optimization problem can be converted into a static quadratic problem which does not require much computational effort and usually yields a global optimum. Nonlinear MPC, however, can suffer from non-convexity which in turn complicates the optimization procedure and prolongs the time until convergence of the optimization problem is achieved.

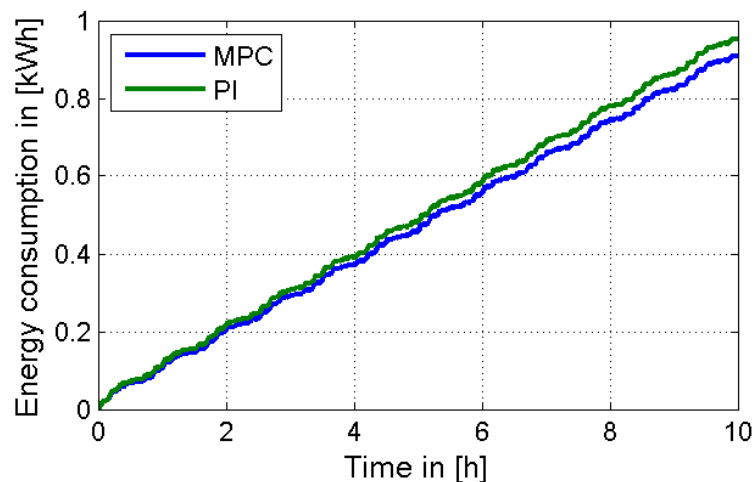

Fig. 3. Savings of electric consumption with MPC (lower curve) versus linear PI-controller (upper curve).

The performance of the linear MPC approach is outlined in Fig. 3 where the energy consumption for a solar thermal HVAC system using centrifugal pumps as system actuators is analyzed. The incorporation of the MPC control concept for a 10 hours daily operation cycle results in a lower electric energy consumption of approx. 5\% in comparison to the conventional PI control. Hereby, the MPC control concept accounts for electric energy savings in the first place. Better performance could be achieved if nonlinear MPC is employed where the solar power gains are included in the optimization routine as well. In that case the control goal is not aiming at stabilization of certain temperature levels or desired values but at the maximization of available energy gains for storage.

\section{CONCLUSION}

The combination of simulation and controls offers new possibilities in building automation. All the necessary prerequisites are available: buildings with intrinsic thermal storages, building automation for fine-grained control, modeling simulation tools for thermal simulation and the algorithms to optimize building operation in order to achieve increased energy efficiency, grid friendliness or other optimization goals. The three methods shown in this paper have different levels of complexity with regard to modeling. All three can increase the overall energy efficiency. While the first method gives a coarse worst-case estimation on how long an HVAC system can be suspended from operation before leaving acceptable indoor comfort levels, the second method provides a general way of optimizing control strategies in building automation. Finally, using MPC is the next logical step in building controls, because it enables the controller to foresee system behavior and adapt the control variables not only based on the current deviation between setpoint and the actual value, but based on an optimization of control variables within a defined prediction horizon. All three methods show that today the potential in increasing energy efficiency by means of building automation is available and can be exploited with some additional engineering effort. The next step has to be a real-world proof of the simulations in order to assess the actual energy savings.

\section{ACKNOWLEDGMENT}

The authors would like to thank the Federal Government of Austria - managed by the Austrian Research Promotion Agency - which made the work on project Building2Grid and KOMBINE possible.

\section{REFERENCES}

[1] D. Dietrich, D. Bruckner, G. Zucker, and P. Palensky, "Communication and computation in buildings: A short introduction and overview," Industrial Electronics, IEEE Transactions on, vol. 57, no. 11, pp. 3577-3584, 2010.

[2] G. Zucker and C. Hettfleisch, "Using simulation for optimized building operation," Proceedings of the e-nova 2010, 2010.

[3] EN 15232:2007 (draft): Energy performance of buildings Impact of Building Automation, Controls and Building Management, CEN/TC247 91.140.10 (Central heating systems) Std., 2011.

[4] D. Basciotti, F. Judex, O. Pol, and R.-R. Schmidt, "Sensible heat storage in district heating networks: a novel control strategy using the network as storage," 6th International Renewable Energy Storage Conference and Exhibition (IRES 2011), November 2011.

[5] A. A. Zaidi, F. Kupzog, T. Zia, and P. Palensky, "Load recognition for automated demand response in microgrids," in Proceedings of the 36th IEEE Conference on Industrial Electronics, IECON 2010, Phoenix AZ, USA, 2010.

[6] P. Palensky and D. Bruckner, "Anticipative virtual storage power plants," in Proceedings of IEEE IECON 2009, Porto, Portugal, 2009. 

fähig?" in Tagungsband der ComForEn 2010, vol. OVE Schriftenreihe Nr. 57, 2010, pp. 24-26.

[8] TRNSYS 17 - a TRaNsient SYstem Simulation program, Solar Energy Laboratory, Univ. of Wisconsin-Madison, 2010. [Online]. Available http://www.transsolar.com/_software/download/en/trnsys_shortinfo_en.pdf [9] B. D. B. Crawley and L. K. Lawrie, "Energyplus: Energy simulation program," Manager, vol. 42, no. 4, pp. 49-56, 2000.

[10] P. Fritzson, Introduction to modeling and simulation of technical and physical systems with Modelica. Hoboken, N.J: Wiley IEEE Press, 2011.

[11] "Dymola - multi-engineering modeling and simulation," 2011 [Online]. Available: www.dymola.com

[12] G. Zucker，R. Braun，F. Judex，and C. Hettfleisch, "Worst-case abschätzung von thermischem gebäudeverhalten," in Proceedings of the enova 2011, 2011 .

[13] P. Palensky, G. Zucker, F. Judex, R. Braun, F. Kupzog, T. Gamauf, and J. Haase, "Demand response with functional buildings using simplified process models," in Proceedings of the 37th Annual Conference of the IEEE Industrial Electronics Society (IECON 2011), 2011, pp. 31133118

[14] "Google SketchUp - 3D modeling for everyone," 2011. [Online]. Available: http://sketchup.google.com

[15] IEC 61131-3 - Programmable controllers - Part 3: Programming languages, International Electrotechnical Commission (IEC) Std., 2003.

[16] J. Siroky, F. Oldewurtel, J. Cigler, and S. Privara, "Experimental Analysis of Model Predictive Control for an Energy Efficient Building Heating System," Applied Energy, vol. 88, pp. 3079-3087, Apr. 2011 [Online]. Available: http://control.ee.ethz.ch/index.cgi?page= publications; action $=$ details; $i d=3818$

[17] D. Gyalistras, M. Gwerder, F. Oldewurtel, C. Jones, M. Morari, B. Lehmann, K. Wirth, and V. Stauch, "Analysis of energy savings potentials for integrated room automation," in 10th REHVA World Congress, Sustainable Energy Use in Buildings-CLIMA 2010. Antalya, Turkey, 2010.

[18] F. Oldewurtel, D. Gyalistras, M. Gwerder, C. N. Jones, A. Parisio, B. Lehmann, and M. Morari, "Increasing energy efficiency in building climate control using weather forecasts and model predictive control," in Clima - RHEVA World Congress, Antalya, Turkey, May 2010. [Online]. Available: $\quad \mathrm{http}: / /$ control.ee.ethz.ch/index.cgi?page= publications;action= details;id $=3545$

[19] E. Camacho and C. Bordons, Model predictive control. Springer Verlag, 2004.

[20] C. Hettfleisch, F. Dubisch, S. Ledinger, G. Zucker, and P. Palensky, "Energieeinsparpotential eines Passivhauses unter Berücksichtigung von Wetterprognosen," Bauphysik, vol. 6, pp. 399-404, December 2010. 